\title{
Color diagnosticity in object recognition
}

\author{
JAMES W. TANAKA \\ Oberlin College, Oberlin, Ohio \\ and \\ LYNN M. PRESNELL \\ College of Wooster, Wooster, Ohio
}

\begin{abstract}
Does color influence object recognition? In the present study, the degree to which an object was associated with a specific color was referred to as color diagnosticity. Using a feature listing and typicality measure, objects were identified as either high in color diagnosticity or low in color diagnosticity. According to the color diagnosticity hypothesis, color should more strongly influence the recognition of high color diagnostic (HCD) objects (e.g., a banana) than the recognition of low color diagnostic (LCD) objects (e.g., a lamp). This prediction was supported by results from classification, naming, and verification experiments, in which subjects were faster to identify color versions of HCD objects than they were to identify achromatic versions and incongruent color versions. In contrast, subjects were no faster to identify color versions of LCD objects than they were to identify achromatic and incongruent color versions. Moreover, when shape information was degraded but color information preserved, subjects were less impaired in their recognition of degraded HCD objects than of degraded LCD objects, relative to their nondegraded versions. Collectively, these results suggest that color plays a role in the recognition of HCD objects.
\end{abstract}

Work in the neurophysiology of vision has revealed that, very early on in processing, the human visual system segregates stimulus input according to separate perceptual dimensions, such as brightness, movement, color, and depth (Livingstone \& Hubel, 1987). Psychological models of visual processing have postulated that these dimensions serve an important function in defining the shape of the visual stimulus (Cavanagh, 1987). An important question is whether these same dimensions might contribute to later stages of visual processing that are involved in the recognition of the stimulus input. In this paper, we examine the potential contribution of one of these dimensions, color, and consider the role that color might play in object recognition.

In vision research, a distinction is often made between the processes of early vision and the processes of late vision (Marr, 1982; Neisser, 1967; Ullman, 1984). Early visual processes are crucial for registering and grouping visual input according to common properties. In Marr's

This research was supported by NIH Grant R15 HD30433, a Keck Foundation Faculty Research Award, and a COSEN summer research grant. We thank Jen Sable and Michael Giles for their assistance in conducting this research and Marjorie Henderson for her help in preparing this manuscript. We are grateful to Cleve Gilmore for the use of his photometer and technical assistance. Gary Gillund and Jim Danemiller provided useful comments on an earlier version of the paper. We would also like to acknowledge Mike Braunstein, Glyn Humphreys, Tom Sanocki, and an anonymous reviewer for their helpful suggestions and advice. Correspondence concerning this article should be addressed to J. Tanaka, Department of Psychology, Severance Lab, Oberlin College, Oberlin, OH 44074 (e-mail: tanaka@cs.oberlin.edu).

-Accepted by previous editor, Myron L. Braunstein primal sketch, for example, the incoming stimulus is organized into blobs, bars, corners, and edges, which are essential for establishing an object's three-dimensional surface and shape properties. In this regard, color contributes to the initial segmentation of an object by grouping areas of the visual input according to the dimension of color (Callaghan, 1984; Legge, Parish, Luebker, \& Wurm, 1990; Troscianko \& Harris, 1988).

Late visual processes, on the other hand, are responsible for the processes of object recognition, in which the segmented object is matched to a representation stored in memory. Is color information important in object recognition? Proponents of edge-based theories claim that objects are initially recognized solely on the basis of their shape (Biederman, 1987; Grossberg \& Mingolla, 1985). According to these theories, the representations mediating initial object recognition contain information about an object's shape but contain no information about an object's color or texture. In contrast, surface-plus-edge-based theories propose that surface information, such as color and texture, can be used, in conjunction with shape information, to facilitate initial object recognition (Gibson, 1969). According to surface-plus-edge-based theories, object representations include information not only about an object's shape, but also about an object's color and texture.

Experiments intended to test the competing claims of edge-based and surface-plus-edge-based theories have yielded equivocal results. For example, several studies have shown that subjects are no faster to recognize appropriately colored objects (e.g., a yellow banana) than they are to recognize gray-scale versions (Biederman \& Ju, 1988; Davidoff \& Ostergaard, 1988; Ostergaard \& Davidoff, 1985). Other studies, in which the question of whether in- 
consistent color information interferes with object recognition processes has been examined, have shown that subjects are no slower to recognize inappropriately colored objects (e.g., a purple banana) than they are to recognize appropriately colored objects (Ostergaard \& Davidoff, 1985). The demonstrated failure of color to produce either a facilitative or an interference effect on recognition suggests that it plays little or no role in object recognition processes. On the basis of this evidence, some researchers have concluded that normal recognition must be based on shape information alone (Biederman, 1987; Grossberg \& Mingolla, 1985).

In contrast with studies reporting a null effect of color, other studies have demonstrated a reliable effect of color information on object recognition. For example, several studies have shown that appropriately colored objects are recognized faster than monochrome objects (Davidoff \& Ostergaard, 1988; Humphrey, Goodale, Jakobson, \& Servos, 1994; Price \& Humphreys, 1989; Wurm, Legge, Isenberg, \& Luebker, 1993) and inappropriately colored objects (Humphrey et al., 1994; Joseph \& Proffitt, 1996; Price \& Humphreys, 1989). Moreover, neuropsychological studies have shown that brain-injured patients with impaired object recognition abilities demonstrate improved performance when identifying colored objects, rather than black and white and gray-scale images (Humphrey et al., 1994; Mapelli \& Behrmann, 1997). Consistent with the surface-plus-edge-based theory of recognition, these results suggest that color information can be a useful source of information in object recognition.

Why might some studies find evidence supporting the effects of color on object recognition processes, whereas other studies do not? The extent to which color influences object recognition might depend on an object's color diagnosticity. Color diagnosticity refers to the degree to which a color is associated with or symptomatic of a particular object. For example, whereas the color red might be diagnostic of the object fire engine, red would not be diagnostic of the object car. According to the color diagnosticity hypothesis, color information would affect recognition of objects that are high in color diagnosticity but would not affect the recognition of objects that are low in color diagnosticity.

The goal of the present experiments was to test the predictions of the color diagnosticity hypothesis. Using a feature-listing and typicality task, objects that were strongly associated with a particular color were identified as high color diagnostic (HCD) objects. In contrast, objects that were weakly associated with color were identified as low color diagnostic (LCD) objects. Subjects identified achromatic and chromatic versions of HCD and LCD objects in classification, naming, and verification experiments. The main finding of these experiments was that, whereas color information influenced the recognition of HCD objects, it did not affect the recognition of LCD objects. Consistent with the color diagnosticity hy- pothesis, these results suggest that color plays a role in the recognition of objects with strong color associations.

\section{EXPERIMENT 1 \\ Feature Listing and Typicality Judgments}

The question of color diagnosticity and object recognition has been addressed by several researchers (Biederman \& Ju, 1988; Wurm et al., 1993). In the study by Biederman and Ju, 29 objects were rated by a panel of three judges as to whether or not color was diagnostic for that object. They predicted that, if color was contributing to object recognition, objects for which color is diagnostic should benefit more from the inclusion of color information than should those objects for which color was judged to be nondiagnostic. However, the results of a series of five experiments revealed that the presence of color information did not facilitate object recognition for either the color diagnostic or the color nondiagnostic objects. In another study, Wurm et al. found that color information facilitated the overall recognition of food items. However, they reported that the magnitude of the color facilitation did not correlate with the measure of color diagnosticity.

Although previous studies failed to identify color diagnosticity as a factor in object recognition, it is important to examine the issue of color diagnosticity through the collection of normative data. In Experiment 1, color diagnosticity was assessed with two measures: feature listing and typicality judgments. In the feature-listing task, the subjects listed perceptual features that were associated with the target object. In the typicality task, the subjects were asked to indicate the color that was most typical of the target object. An object was rated as high in color diagnosticity if a specific color was consistently mentioned first in the feature list and was rated as the typical color of the object.

As measures of color diagnosticity, the feature-listing and typicality ratings differed from the cue validity measure employed by Wurm et al. (1993). In their study, subjects were provided with a color name and rated the relative symptomaticity of the color for 20 food items. An object was rated as high in color diagnosticity if a color was highly symptomatic of one food object and not symptomatic of other food objects. Feature listing and typicality judgments, rather than the cue validity measure, were used because objects tested in the present study were drawn from a variety of artifactual and natural categories.

\section{Method}

Subjects. The 30 subjects were students enrolled at Oberlin College. None of the subjects had participated in the previous color studies. All the subjects reported normal or corrected-to-normal vision and normal color vision. The subjects received class credit for their participation.

Materials. The 48 object names selected for the feature-listing task of Experiment I are Iisted in Appendix A. One half of the object names represented natural (biological) categories, and the re- 
Table 1

High and Low Color Diagnostic Objects, Based on the Percentage of Subjects Who Agreed on the Object's Typical Color (Shown in Parentheses) and Mentioned Typical Color First in Feature List

\begin{tabular}{|c|c|c|}
\hline Objects & $\%$ Listed as Typical & $\%$ Listed First \\
\hline \multicolumn{3}{|c|}{ High Color Diagnostic } \\
\hline Taxi & 100 (yellow) & 97 \\
\hline Fire engine & 100 (red) & 97 \\
\hline Lemon & 100 (yellow) & 90 \\
\hline Lime & 100 (green) & 87 \\
\hline Radish & 83 (red) & 83 \\
\hline Banana & 100 (yellow) & 83 \\
\hline Lettuce & 97 (green) & 83 \\
\hline Broccoli & 100 (green) & 83 \\
\hline Stop sign & $100(\mathrm{red})$ & 80 \\
\hline Corn & 100 (yellow) & 80 \\
\hline Carrot & 100 (orange) & 80 \\
\hline Brick & 87 (red) & 80 \\
\hline \multicolumn{3}{|c|}{ Low Color Diagnostic } \\
\hline Table & 100 (brown) & 0 \\
\hline Dog & 80 (brown) & 0 \\
\hline Chair & 90 (brown) & 0 \\
\hline Hammer & 66 (silver) & 3 \\
\hline Fish & 50 (silver) & 3 \\
\hline Bird & 23 (brown) & 3 \\
\hline Saw & 77 (silver) & 3 \\
\hline Lamp & 53 (white) & 7 \\
\hline Nail & 83 (silver) & 10 \\
\hline Screwdriver & 83 (silver) & 13 \\
\hline Fork & 87 (silver) & 23 \\
\hline Sportscar & 100 (red) & 23 \\
\hline
\end{tabular}

maining object names represented artifactual (human-made) categories. All of the items were basic level categories (e.g., dog, banana, fork). The majority of the objects included in Experiment 1 had already been utilized as stimuli in other studies of color and object recognition (Biederman \& Ju, 1988; Davidoff \& Ostergaard, 1988; Ostergaard \& Davidoff, 1985; Price \& Humphreys, 1989). Additional common objects were also chosen for inclusion in the feature-listing task on the basis of their high typicality, as ranked by Battig and Montague's (1969) category norm study. Object names were printed at the top of separate sheets of $3 \times 5$ in. paper and randomly assembled into test booklets, with the restriction that object names sharing the same superordinate category (natural or artifactual) could not appear in consecutive presentations.

Procedure. The experiment was divided into two parts. In the first part, the subjects were given a booklet consisting of 48 object names, with each name written at the top of the page. The subjects were told that, at the experimenter's signal, they would be given $10 \mathrm{sec}$ to list three perceptual features that described the object. For example, the subjects were told if the object name was dime, they could list perceptual features such as round, shiny, and small. The 10 -sec time limit was imposed in order to access the subjects' primary perceptual impressions of each object and to avoid listings of more conceptual or functional qualities. After the subjects completed the feature-listing part of the experiment, they were instructed to list the typical color for each object. The subjects were tested in groups.

\section{Results and Discussion}

The subjects' feature lists were scored for the presence/ absence of a color feature and its rank. For example, if a subject had listed the features for banana as yellow, smooth, and long (in that order), the color feature yellow would have been tallied under the first rank position for the color yellow on the banana score sheet. The color listed and the rank at which it was listed (first, second, third) were noted and tallied for each object.

Color diagnosticity was determined on the basis of feature-listing and typicality responses. The objects were rank-ordered according to the percentage of subjects that mentioned a color as the first feature in the feature lists (see Appendix A). The top 12 objects in the rank ordering were identified as HCD objects if the same color was indicated as the typical color by at least $80 \%$ of the subjects. The $12 \mathrm{HCD}$ objects were taxi, fire engine, lemon, lime, radish, brick, banana, lettuce, broccoli, stop sign, corn, and carrot. The LCD objects were objects for which a color was rarely or never mentioned as the first feature in the feature list. The $12 \mathrm{LCD}$ objects were table, dog, chair, hammer, fish, bird, saw, lamp, nail, screwdriver, fork, and sportscar (see Table 1). Of the seven items (apple, banana, fish, nail, fork, flowerpot, and camera) considered to be high in color diagnosticity by Biederman and Ju (1988), only banana was identified as being high in color diagnosticity according to the combined featurelisting and typicality measures. Apple, flowerpot, and camera were moderately associated with a particular color. Interestingly, objects identified as HCD in the Biederman and Ju study (i.e., fish, nail, and fork) were rated as LCD objects in the present study.

Why might the same object be identified as HCD in the Biederman and Ju (1988) study and as LCD in the present study? An important difference between the two studies was the method by which color diagnosticity was determined. Whereas the present study employed featurelisting and typicality responses to determine color diagnosticity, Biederman and Ju selected objects on the basis of their typicality alone. As measures of color diagnosticity, typicality judgments and feature-listing responses were only weakly correlated $(r=.543)$. For example, although all of the subjects in Experiment 1 agreed that brown was the typical color for chair, none of them mentioned brown as one of the top three perceptual characteristics in their feature lists, and thus, chair was classified as low in color diagnosticity. In feature listing, the subjects seemed to list only those perceptual characteristics that differentiate the target object from its contrast categories. In the above example, although the color brown is typical of chair, it may not be very informative with regard to distinguishing it from other category members, such as table or sofa. On the other hand, the color yellow was frequently mentioned in feature lists for $b a$ nana, because yellow is useful for distinguishing a banana from other members of the fruit category. The combined feature-listing and typicality measure is similar to a cue validity approach in which the information value of the cue is determined with respect to its total frequency within a category in proportion to the frequency with which it occurs in other contrasting categories (Rosch, Mervis, Gray, Johnson, \& Boyes-Braem, 1976). Whether the feature-listing approach is a useful predictor of how 
color affects object recognition performance is examined in the next experiment.

\section{EXPERIMENT 2 Object Classification Task}

In Experiment 1, HCD and LCD objects were identified with feature-listing and typicality measures. In Experiment 2 , the effects of color diagnositicity on recognition were tested in an object classification task. In object classification, two object labels are simultaneously presented on a computer screen to the left and right of midline. After a short delay, the stimulus object appears in the center of the screen. The subject indicates whether the object matches the label on the left or on the right by pressing the corresponding response key.

The object classification task is a useful paradigm for studying object recognition for several reasons. First, in object classification, the foil object that serves as the contrast category for the target object can be explicitly manipulated (Price \& Humphreys, 1989). In the present study, the selected foil objects shared the same semantic superordinate category as the target object, but differed from the target object with respect to their color and shape. Second, similar to other object verification tasks, in object classification, the lexical name activates the object representation that is subsequently matched against the picture stimulus. With this type of paradigm, if color information is stored as part of an HCD object representation, a color picture stimulus should serve as a better match to its activated object representation than would a noncolor picture stimulus (Biederman \& Ju, 1988).

According to the color diagnosticity hypothesis, color should more strongly influence the recognition of HCD objects than the recognition of LCD objects (e.g., a lamp). Specifically, subjects should be faster to classify $\mathrm{HCD}$ objects when presented in color than in gray scale. In contrast, subjects should be no faster to classify color versions of LCD objects than to classify gray-scale versions. On the other hand, if surface color plays no role in object recognition, subjects should show no difference when recognizing chromatic and achromatic versions of either HCD or LCD objects.

\section{Method}

Subjects. The 45 subjects were students enrolled at Oberlin College. None of the subjects had participated in the previous experiment. All the subjects reported normal or corrected-to-normal vision and normal color vision. The subjects received class credit for their participation.

Stimuli. The stimuli consisted of 48 pictures of common objects. As determined by the ratings reported in Experiment 1, 12 objects were considered HCD, and 12 were rated as low in color diagnosticity. Each of the HCD and LCD objects was paired with a foil object. Foil objects were selected on the basis of being distinct in shape and color from the target objects and were members of the same superordinate category (e.g., fruit, vegetable, or vehicle). It was also important that the targets and foils be of similar size. Judgments of typicality were also employed in the foil object selection and were based on category norms collected by Battig and Mon- tague (1969). It was necessary that the foil objects be at least fairly typical. Final judgments on the choice of foils were reviewed by a panel of three experimenters. A list of the 24 target objects and their respective foils is presented in Appendix B. Pictures of each of the 24 target and 24 foil objects were obtained from the MacMillan $\mathrm{Vi}$ sual Dictionary (Corbeil \& Archambault, 1992). They were digitized, using a MicroTek scanner, and prepared for presentation through the use of the Adobe Photoshop graphics program.

Each picture was presented in two versions: a color version and an achromatic, gray-scale version. The color versions of the object pictures consisted of pictures of the objects in the colors in which they naturally or typically appear. Each picture contained the object displayed against a neutral white background. A Spectra Pritchard photometer (Model 1980-A) was used to compute luminance values for each image. A photopically filtered aperture of $1^{\circ}$ of visual angle was positioned at the center of each image, and the mean luminance of this area was recorded. The mean luminance values for HCD images displayed in gray scale and color were 12.52 and $12.94 \mathrm{~cd} / \mathrm{m}^{2}$, respectively. The mean luminance values for LCD images displayed in gray scale and color were 12.81 and $13.12 \mathrm{~cd} / \mathrm{m}^{2}$, respectively. (See Appendix $\mathrm{C}$ for the luminance values of individual objects.)

Object images subtended an averaged visual angle of $5.9^{\circ}$ in the horizontal dimension and $4.7^{\circ}$ in the vertical dimension. To minimize picture familiarity effects, pictures were shown in different left/right orientations in the achromatic and color conditions. The labels for the 24 target and foil object pairs (e.g., radish-onion) were printed in an 18-point font. For half the trials, the target name was listed on the left side of the screen and the foil name on the right side of the screen, and for the other half of the trials, the order was reversed.

Procedure. All the experiments described in this paper were conducted on Macintosh computers using the SuperLab experimental software package. In Experiment 2, the subjects saw two object names on the computer screen, one on the left side of the screen and one on the right for $2.500 \mathrm{msec}$. An object was then presented in the center of the screen above the two names. If the picture matched the name on the left side of the screen, the subject was to press the key marked "left" (" 4 " on the numeric keypad). If the picture matched the name on the right side of the screen, the subject was to press the key marked "right" (" 6 " on the numeric keypad). Reaction time was measured from the onset of the stimulus picture. The stimulus picture remained on the screen until the subject responded. The subjects were instructed to respond as quickly as possible. The 48 target objects and 48 foil objects were presented once in an achromatic and color display. Thus, there were 192 total trials. Trials were randomized across subjects.

\section{Results and Discussion}

Results were calculated only on the trials in which the target items were presented; trials in which the foil items appeared were not included in these analyses.

Accuracy. An analysis of variance (ANOVA) test was performed for the accuracy data, with display (color, gray scale) and color diagnosticity (high, low) as withinsubjects factors. The main effect of display was significant $\left[F(1,44)=10.56, M S_{\mathrm{e}}=4.36, p<.05\right]$. The subjects made fewer errors when categorizing color object pictures $(M=3 \%)$ than when categorizing achromatic pictures $(M=5 \%)$. The main effect of color diagnosticity was also significant $\left[F(1,44)=7.97, M S_{\mathrm{e}}=3.76, p<.05\right]$. The subjects made fewer errors when categorizing LCD object pictures $(M=3 \%)$ than when categorizing HCD pictures $(M=5 \%)$. There was a significant interaction between display and color diagnosticity $\left[F(1,44)=10.50, M S_{\mathrm{e}}=\right.$ 


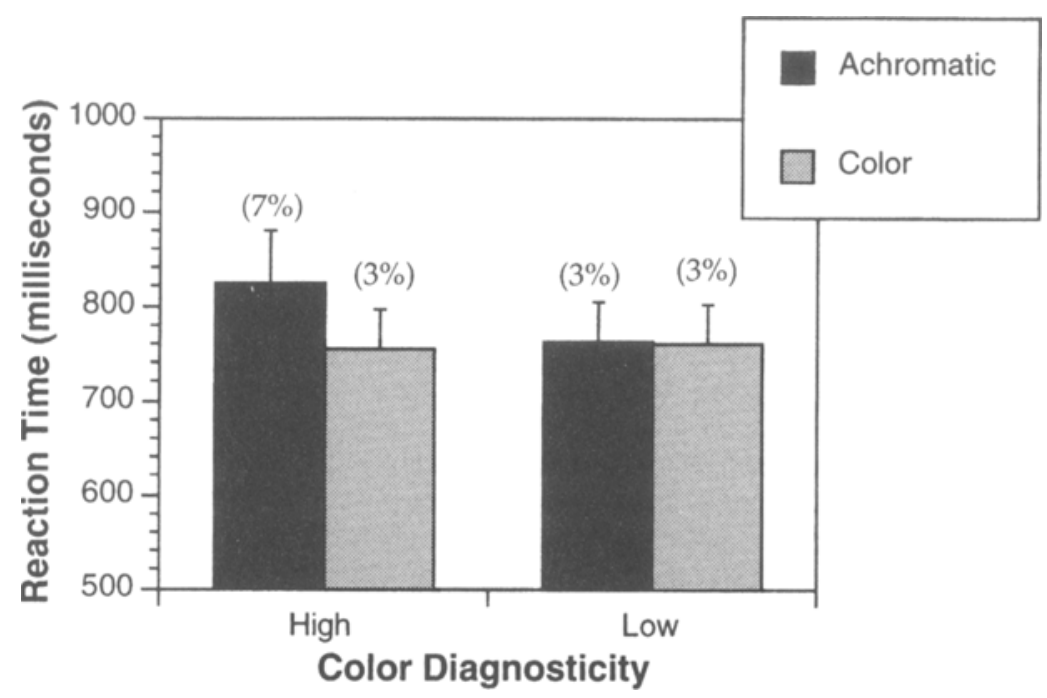

Figure 1. Mean reaction times for object classification (Experiment 2) as a function of color diagnosticity and color.

$3.76, p<.05]$. The subjects were less accurate in categorizing the achromatic versions of the HCD items than they were in categorizing the color versions of those same items. Accuracy levels remained consistent across picture conditions for the LCD objects.

Reaction time. An ANOVA was performed on the reaction time data from the correct trials with display (color, gray scale) and color diagnosticity (high, low) as withinsubjects factors. The main effect of display was not significant $\left[F(1,44)=3.33, M S_{\mathrm{e}}=59,800, p>.05\right]$. Overall, the subjects recognized achromatic objects $(M=794 \mathrm{msec})$ as quickly as color objects $(M=757 \mathrm{msec})$. Moreover, there was no main effect of diagnosticity $[F(1,44)=1.96$, $\left.M S_{\mathrm{e}}=31,174, p>.05\right]$, so that the subjects were no faster to match HCD objects $(M=789 \mathrm{msec})$ to their object names than they were to match LCD objects $(M=$ $762 \mathrm{msec}$ ). However, there was a significant interaction between color diagnosticity and display $[F(1,44)=4.07$, $\left.M S_{\mathrm{e}}=51,150, p=.05\right]$. As can be seen in Figure 1, whereas the subjects were more efficient at categorizing color pictures than at categorizing achromatic pictures of HCD objects, they showed no difference in classifying color pictures and achromatic pictures of LCD objects.

Tukey post hoc comparisons revealed that the difference between the achromatic and the chromatic conditions was significant for HCD objects $(p<.05)$, but not for LCD objects $(p>.05)$. The difference in reaction time scores between HCD and LCD objects in the achromatic condition was not significant.

An ANOVA was performed, with items as the random factor and display and color diagnosticity as withinitems factors. The main effects of display and color diagnosticity were not significant; however, the critical interaction of color diagnosticity and display was found to be significant $\left[F(1,23)=7.23, M S_{\mathrm{e}}=604,466, p<.05\right]$. Inspection of the individual reaction times of the target ob- jects listed in Appendix D shows that all of the HCD objects were facilitated by the presence of color information in the display. However, contrary to the color diagnostic hypothesis, some of the LCD objects (e.g., table, screwdriver) also benefited from the presence of color in the display.

In summary, general predictions of the color diagnosticity hypothesis were supported in Experiment 2, in which color facilitated recognition of HCD objects but had little effect on the recognition of LCD objects. The color diagnosticity findings differ from previously reported null findings in which the magnitude of the color advantage was not correlated with the color diagnosticity of the object (Wurm et al., 1993). Owing to the their null color diagnosticity effect, Wurm et al. concluded that color influences early stages of object processing involved in object and feature segregation but does not affect the later stage of object recognition. However, only food objects were tested in the Wurm et al. study, and the majority of these objects (e.g., carrot, apple) are presumably high in color diagnosticity. It is possible that a wider range of HCD and LCD objects might have produced a correlation between color diagnosticity and color effects. The results of Experiment 2 indicate that color effects vary as a function of an object's color diagnosticity when a range of HCD and LCD objects is tested.

\section{EXPERIMENT 3 Naming Study}

The main finding of Experiment 2 was that color influences the recognition of $\mathrm{HCD}$ objects but does not affect the recognition of LCD objects. However, there is an alternative explanation for these results. It is conceivable that the subjects employed a special color-first strategy for the recognition of HCD objects. For example, sup- 
pose that a subject in Experiment 2 sees the word label banana-cucumber and adopts the strategy of if yellow, then banana. If the color version of the banana appears, the subject will be quick to respond banana. On the other hand, if the achromatic version of the banana appears, the subject will be forced to rely on a secondary shape strategy, and response time will be slowed. According to this account, subjects could bypass shape information altogether when identifying color versions of HCD objects. Of course, subjects could not use the color-first strategy for recognizing LCD objects, because color is not informative for recognition of these objects. This explanation fits the pattern of results obtained in Experiment 2, in which HCD objects (color-first strategy) were recognized more quickly than achromatic HCD objects (switch to shape-only strategy) and in which no difference was found between color and achromatic LCD objects (shape-only strategy).

The alternative color-first hypothesis was tested in the present experiment with a naming paradigm. In a naming task, the subject vocally responds to a presented object with its corresponding lexical term. Naming is a good test of this alternative explanation, because subjects cannot anticipate the stimulus prior to its presentation. Therefore, they would be unable to use a color-first strategy. If color diagnosticity were an artifact of the color-first strategy in the previous object classification study, no color diagnosticity effects would be expected in the present naming study. On the other hand, if color diagnosticity influences subjects' abilities to name objects, naming latencies would be faster for chromatic versions of HCD objects, relative to achromatic versions, and no difference should be found between the naming latencies for color and achromatic versions of LCD objects.

\section{Method}

Subjects. The subjects were 36 students from Oberlin College. None of the subjects had participated in the previous experiments. All the subjects reported normal or corrected-to-normal vision and normal color vision. The subjects received class credit for their participation.

Stimuli. The 24 HCD and LCD pictures described in Experiment 2 were used as stimuli in the present study. Images subtended an average visual angle of $5.9^{\circ}$ in the horizontal dimension and $4.7^{\circ}$ in the vertical dimension. Similar to Experiment 2, images were shown in different left/right orientations in their achromatic and color versions. Naming latencies were measured with a voice-activated relay switch connected to a National Instruments (NB-DIO-24) card with a temporal resolution of $1 \mathrm{msec}$.

Procedure. The subjects were instructed that they would see pictures of common objects and that their task was to name the objects as quickly and accurately as they could. Prior to beginning the experiment, the subjects read over a list of the 24 stimulus objects. The subjects were seated $50 \mathrm{~cm}$ in front of the computer screen. At the beginning of each experimental trial, the word READY appeared on the computer screen for $2 \mathrm{sec}$. The READY prompt was followed by the stimulus picture. The picture remained on the screen until the subject made a response. The subjects named both achromatic and color versions of the objects. The achromatic and color versions of the 24 objects produced a total of 48 experimental trials. The ex- perimental trials were randomly presented across subjects. The subjects were tested individually.

Naming responses were recorded by one of the experimenters, who was seated directly behind the subject. A response was scored as incorrect if the subject could not identify the object or if the name provided was at a level of abstraction that was more general than the target name (e.g., the name fruit given for the picture banana). Names that were at a more specific level than was the target name were scored as correct (e.g., the name robin given for the picture bird).

\section{Results and Discussion}

Accuracy. An ANOVA was performed for the accuracy data, with display (color, achromatic) and diagnosticity (high, low) as within-subjects factors. Overall, the subjects committed fewer naming errors for $L C D$ objects $(M=2 \%)$ than for HCD objects $[M=9 \% ; F(1,35)=$ 73.76, $\left.M S_{\mathrm{e}}=33.06, p<.05\right]$ and fewer errors naming color versions of objects $(M=3 \%)$ than naming achromatic versions $\left[M=7 \% ; F(1,35)=19.53, M S_{\mathrm{e}}=8.51\right.$, $p<.05]$. The interaction between display and diagnosticity was also significant $\left[F(1,35)=15.83, M S_{\mathrm{e}}=5.84\right.$, $p<.05]$. As post hoc comparisons revealed, the subjects were significantly less accurate in naming achromatic versions of HCD objects than in naming chromatic versions $(p<.05)$. In contrast, no difference was found between the naming accuracy of color and gray-scale versions of LCD objects $(p>.05)$.

Naming latencies. Naming latencies that were three standard deviations above the mean were scored as errors and were excluded from the reaction time analysis. For the correct trials, an ANOVA was performed, with color (achromatic, color) and color diagnosticity (high, low) as within-subjects factors. As is shown in Figure 2, subjects were faster to name LCD objects $(M=694 \mathrm{msec})$ than to name HCD objects $[M=775 \mathrm{msec} ; F(1,35)=62.81$, $\left.M S_{\mathrm{e}}=233,128, p<.05\right]$, but overall, the subjects were no faster to name color objects $(M=727 \mathrm{msec})$ than to name achromatic objects $[M=742 \mathrm{msec} ; p>.05]$. However, display interacted with diagnosticity $[F(1,35)=10.05$, $\left.M S_{\mathrm{e}}=19,956, p<.005\right]$. Post hoc comparisons showed that color versions of HCD objects were named significantly faster than their achromatic versions $(p<.05)$. In contrast, achromatic versions of LCD objects were named as quickly as color versions.

An ANOVA was completed, with items as the random factor and display and color diagnosticity as withinitems factors. Although there was a reliable difference between HCD and LCD objects $\left[F(1,22)=9.56, M S_{\mathrm{e}}=\right.$ $109,921, p<.01]$, there was no overall difference in display $\left[F(1,22)=1.18, M S_{\mathrm{e}}=2,836, p>.10\right]$. The interaction between diagnosticity and display was significant $\left[F(1,22)=4.20, M S_{\mathrm{e}}=10,121, p=.05\right]$.

To summarize, in Experiment 3, the subjects named HCD objects faster and with less error when shown in color than when shown in gray scale. In contrast, the subjects' naming latencies and errors were the same for LCD objects, whether shown in color or in gray scale. Because in naming, subjects cannot anticipate the stimulus prior 


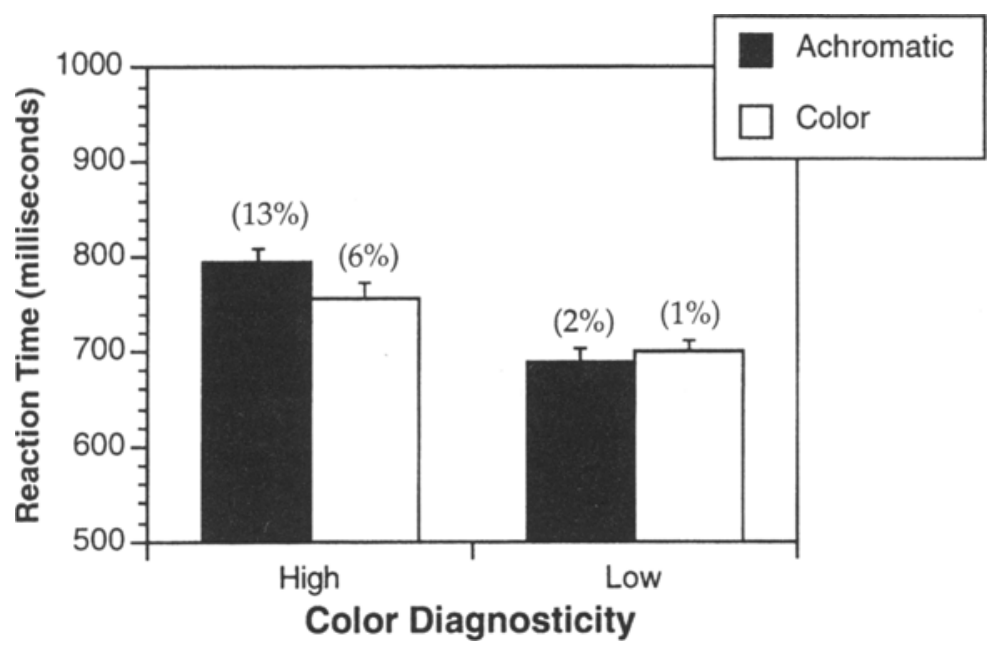

Figure 2. Mean reaction times in naming (Experiment 3) as a function of color diagnosticity and color.

to its presentation, these results seem to rule out the strategic color-first explanation of the color diagnosticity effect found in Experiment 2. However, the naming paradigm produced latencies that were more variable than the classification times reported in the previous experiment. Some HCD objects (e.g., carrot, corn, lime) demonstrated relatively large color effects, whereas other HCD objects (e.g., lettuce, taxi) showed a slight color disadvantage (see Appendix D for naming latencies). Thus, although the general pattern of results was consistent with the color diagnosticity hypothesis, some objects were the exceptions to the color diagnosticity rule.

\section{EXPERIMENT 4 Shape Versus Color Diagnosticity}

The foregoing results suggest that color plays a role in the recognition of HCD objects, but not in the recognition of LCD objects. However, this interpretation is conflated by possible differences between HCD and LCD objects in their shape diagnosticity (i.e., the extent to which objects are distinguishable on the basis of their shape). If HCD objects are correspondingly low in shape diagnosticity, it is likely that surface information (e.g., color) will be used to disambiguate competing shape-similar objects (Price \& Humphreys, 1989). A measure of an object's shape diagnosticity is its recognizability when displayed achromatically, without the presence of potentially disambiguating color information. Consistent with the shape diagnosticity account in Experiments 2 and 3, subject and item analysis revealed that achromatic HCD objects frequently took longer to recognize than achromatic LCD objects. Therefore, it is not clear whether differences between HCD and LCD objects are attributable to differences in their color diagnosticity per se or whether high color diagnosticity interacts with low shape diagnosticity.
In Experiment 4A, shape diagnosticity was controlled by selecting a revised set of LCD objects whose achromatic recognition was equated with achromatic recognition of HCD objects. After matching HCD and LCD objects with respect to their achromatic recognition, the effects of color on recognition were tested in Experiment 4B. In Experiments 4A and 4B, a verification paradigm, in which a word label is presented prior to the presentation of the object picture, was selected for several reasons. First, the word label used in the verification paradigm should activate only one object representation, as compared with the multiple representations elicited by the pair of word labels used in the previous object classification paradigm (Experiment 2 ). Therefore, the representations mediating recognition of the targeted $H C D$ and LCD objects can be more directly addressed with a verification paradigm. Second, because previous studies (Biederman \& Ju, 1988), using a verification paradigm, failed to find effects of color on recognition, it was important to replicate the color effect in this paradigm.

\section{Experiment 4A \\ Equating Achromatic Recognition of Low Color Diagnostic and High Color Diagnostic Objects}

\section{Method}

Subjects. Twenty-four subjects from Oberlin College participated in Experiment 4A. None of the subjects had participated in the previous experiments. All the subjects reported normal or corrected-to-normal vision and normal color vision. The subjects received class credit for their participation.

Stimuli. The stimuli consisted of achromatic versions of HCD and LCD objects. In addition to the $24 \mathrm{HCD}$ and LCD objects used in Experiments 2 and 3, 12 new LCD objects were included in Experiment $4 \mathrm{~A}$. The new objects were identified as low in color diagnosticity according to the diagnosticity ratings of Experiment 1 or according to the judgments of three independent raters. The new LCD objects were bee, cap, cow, crab, forklift, cigar, shoe, tape dis- 
penser, eggbeater, chicken, and paintbrush. Pictures of the new LCD objects were obtained from the MacMillan Visual Dictionary (Corbeil \& Archambault, 1992) and digitized with a MicroTek scanner. Achromatic versions of the objects were cropped and placed on a white background, using the Adobe PhotoShop program. The same foil objects as those used in Experiment 2 were employed in the present experiment and were paired with the same target stimuli (HCD and LCD objects). Additional foil objects for the new LCD objects were selected so that they were distinct in shape and color from the target objects but similar in physical size. Foils were also drawn from the same superordinate category (e.g., fruit, vegetable, or vehicle) as the target object. Object images subtended an average visual angle of $5.9^{\circ}$ in the horizontal dimension and $4.7^{\circ}$ in the vertical dimension.

Procedure. The subjects were instructed that they would see a plus sign followed by an object name. The object name was presented in the center of the computer screen in 18-point type for $1,500 \mathrm{msec}$. The name label was replaced by an achromatic version of either an HCD or an LCD target or foil object. If the picture matched the name, the subjects were told to press the key marked "true" ("4" on the numeric keypad). If the picture did not match the name, they were to press the key marked "false" " 6 " on the numeric keypad). Reaction time was measured from the onset of the stimulus picture. The stimulus picture remained on the screen until the subject responded. The subjects were instructed to respond as quickly but as accurately as possible. The 36 target stimuli and 36 foil stimuli were shown once, for a total of 72 trials. Trials were randomly presented across subjects.

\section{Results and Discussion}

Reaction times were calculated for correct trials of HCD and LCD target objects (trials involving foil objects were omitted from further analyses). The reaction times for the $24 \mathrm{LCD}$ objects were ranked in descending order, beginning with objects with the longest latencies, to objects with the shortest latencies. The LCD objects with the 12 longest average latencies were forklift, bee, paintbrush, eggbeater, bird, chair, saw, screwdriver, tape dispenser, lamp, crab, and fish (see Appendix D). The mean latency for the 12 LCD objects with the longest reaction times was $764 \mathrm{msec}$, as compared with the mean reaction for the $12 \mathrm{HCD}$ objects of $797 \mathrm{msec}$. The difference in reaction time between the $12 \mathrm{LCD}$ and the $\mathrm{HCD}$ objects was not reliable $[t(23)=1.26, p>.10]$.

The goal of Experiment $4 \mathrm{~A}$ was to identify $12 \mathrm{LCD}$ objects whose achromatic recognition times were comparable with the recognition times of the 12 HCD objects. The goal of Experiment $4 \mathrm{~B}$ was to measure recognition times of these objects presented in their congruent, incongruent, and achromatic color conditions. The color diagnosticity hypothesis predicts that, when shape diagnosticity is taken into account, recognition of HCD objects will nevertheless be influenced by the color condition more than will LCD objects.

\section{Experiment 4B \\ Testing Recognition of High Color Diagnostic and Low Color Diagnostic Objects Equated for Shape Diagnosticity}

\section{Method}

Subjects. Thirty Oberlin College subjects participated in Experiment $4 \mathrm{~B}$. None of the subjects had taken part in the previous ex- periments. All the subjects reported normal or corrected-to-normal vision and normal color vision. The subjects received class credit for their participation.

Stimuli. The stimuli consisted of congruent, incongruent, and achromatic versions of HCD and LCD objects and their foils. With the exception of two HCD objects, the same target and foil object images as those used in Experiment 2 were employed in the present experiment. Owing to their relatively long recognition latencies in Experiment 4A, two of the HCD objects (lime and lettuce) were replaced by two new objects. The new objects (apple and celery) were rated as being high in color diagnosticity, according to the ratings in Experiment 1. Pictures of the new HCD objects and their foils (pear and carrot) were obtained from the MacMillan Visual Dictionary (Corbeil \& Archambault, 1992) and were digitized in color, using a MicroTek scanner. Achromatic versions of the new HCD target and foil objects were created by removing color information from the images while retaining gray-scale values. In the incongruent condition, colors not associated with the 24 target and 24 foil objects (e.g., blue banana) were selected by the experimenters and were assigned to the objects, using the NIH Color Image 1.4 software. Object images subtended an average visual angle of $5.9^{\circ}$ in the horizontal dimension and $4.7^{\circ}$ in the vertical dimension.

Procedure. The object verification task described in Experiment $4 \mathrm{~A}$ was followed in the present experiment. The $24 \mathrm{HCD}$ objects, the 24 LCD objects, and the foil objects were shown in their congruent color, incongruent color, and achromatic conditions for a total of 144 trials. Trials were randomly presented across subjects.

\section{Results and Discussion}

Accuracy. An ANOVA was performed for the accuracy data, with display (congruent, incongruent, achromatic) and diagnosticity (high, low) as within-subjects factors. Overall, the subjects committed fewer errors in verifying $L C D$ objects $(M=2 \%)$ than in verifying $H C D$ objects $\left[M=4 \% ; F(1,35)=5.48, M S_{\mathrm{e}}=0.024, p<.05\right]$. Differences in accuracy were also found between congruent $(M=2 \%)$, incongruent $(M=5 \%)$, and achromatic color conditions $\left[M=3 \% ; F(1,35)=3.16, M S_{\mathrm{e}}=0.012\right.$, $p<.05]$. As post hoc comparisons revealed, significant differences in accuracy were found between the congruent and incongruent color conditions $(p<.05)$. The interaction between display and diagnosticity was not significant.

Reaction time. Reaction times were calculated for correct trials of HCD and LCD target objects (trials involving foil objects were omitted from further analyses). An ANOVA was performed on correct latencies, with display (congruent, incongruent, and achromatic) and diagnosticity (high, low) as within-subjects factors. The overall means for the congruent, achromatic, and incongruent displays were 620,651 , and $686 \mathrm{msec}$, respectively $[F(2,58)=$ 7.40, $\left.M S_{\mathrm{c}}=75.691, p<.01\right]$. The difference between HCD $(M=639 \mathrm{msec})$ and LCD $(M=666 \mathrm{msec})$ objects was significant $\left[F(1,29)=4.79, M S_{\mathrm{e}}=48,742, p<.05\right]$. The interaction between display and diagnosticity was also reliable $\left[F(2,58)=9.05, M S_{\mathrm{e}}=39,047, p<.001\right]$.

Post hoc tests revealed that the difference in verification times between HCD and LCD objects was not significant when these objects were displayed in the achromatic condition $(p>.10)$. However, as is shown in Figure 3, color affected the recognition of HCD objects, so that HCD objects were verified more quickly in the congruent color 


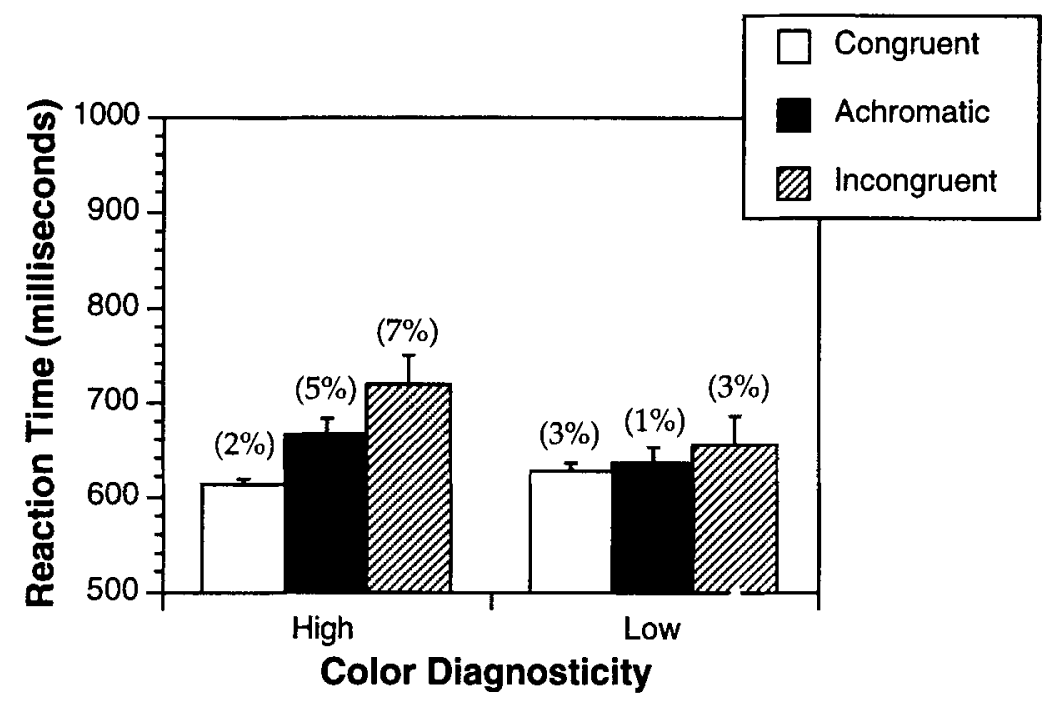

Figure 3. Mean reaction times for object verification (Experiment $4 \mathrm{~B}$ ) as a function of color diagnosticity and color.

condition $(M=612 \mathrm{msec})$ and more slowly in the incongruent color $(M=718 \mathrm{msec})$, relative to the achromatic color condition ( $M=667 \mathrm{msec})$. In contrast, verification times for LCD objects did not reliably differ between the congruent color condition $(M=630 \mathrm{msec})$, the incongruent color condition $(M=662 \mathrm{msec})$, and the achromatic conditions $(M=635 \mathrm{msec}, p>.10)$.

A second ANOVA was completed, with items as the random factor and display and color diagnosticity as withinitems factors. The individual reaction times for the HCD and LCD objects are listed in Appendix D. The item analysis revealed a reliable effect of display $[F(2,22)=$ $\left.8.839, M S_{\mathrm{e}}=28,515, p<.01\right]$ but no overall effect of diagnosticity $\left[F(1,22)=0.973, M S_{\mathrm{e}}=9,800, p>.10\right]$. The interaction between diagnosticity and display approached significance $\left[F(1,22)=2.67, M S_{\mathrm{e}}=8,613, p=.08\right]$.

In an attempt to more closely match achromatic recognition of HCD and LCD objects, the two HCD objects (celery, taxi) with the longest reaction times and the two LCD objects (chair, lamp) with the shortest reaction times were eliminated from the item analysis. After omitting the above objects, the reaction times for verifying achromatic versions of HCD objects ( $M=640 \mathrm{msec}$ ) were slightly faster than the reaction times for verifying achromatic LCD objects $(M=650 \mathrm{msec})$. An ANOVA carried out with the reduced set of HCD and LCD objects showed a reliable effect of display $\left[F(2,18)=11.335, M S_{\mathrm{e}}=\right.$ $30,268, p<.01]$ and no effect of diagnosticity $[F(1,18)=$ $\left.0.063, M S_{\mathrm{e}}=498, p>.10\right]$. The critical interaction between diagnosticity and display reached reliable levels of significance $\left[F(2,18)=4.275, M S_{\mathrm{e}}=11,415, p<.05\right]$.

The purpose of Experiment 4 was to test for color effects of HCD objects that were comparable in their shape information with LCD objects. In Experiment 4A, shape information was controlled by selecting HCD and LCD objects that were roughly equivalent in their achromatic recognition. Experiment $4 \mathrm{~B}$ demonstrated that, whereas color had little effect on the recognition of LCD objects, color either facilitated or interfered with the recognition of HCD objects. These results suggest that color diagnosticity might make a contribution to the recognition of $\mathrm{HCD}$ objects that is independent of information concerning their shape diagnosticity.

\section{EXPERIMENT 5 Degrading Shape Information}

In the foregoing experiments, the effect of color on recognition was investigated by manipulating color information while preserving shape information. The central finding of these experiments was that color information influences the recognition of HCD objects but not the recognition of LCD objects. If color information is not crucial to the identity of LCD objects, recognition must, therefore, be dependent on shape information. In Experiment 5 , the role of shape information on recognition was examined by altering the shape properties of HCD and LCD objects. In this study, the shape properties of HCD and LCD objects were degraded by passing the images through a Gaussian filter. Using this manipulation, the shape properties of the object were changed, but color information was preserved. It was hypothesized that if LCD objects rely on shape information to a greater degree than do HCD objects, they should show a greater impairment in recognition when shape information was degraded, relative to HCD objects. Although shape information is important for the recognition of HCD objects, it may be more important for the recognition of LCD objects. 


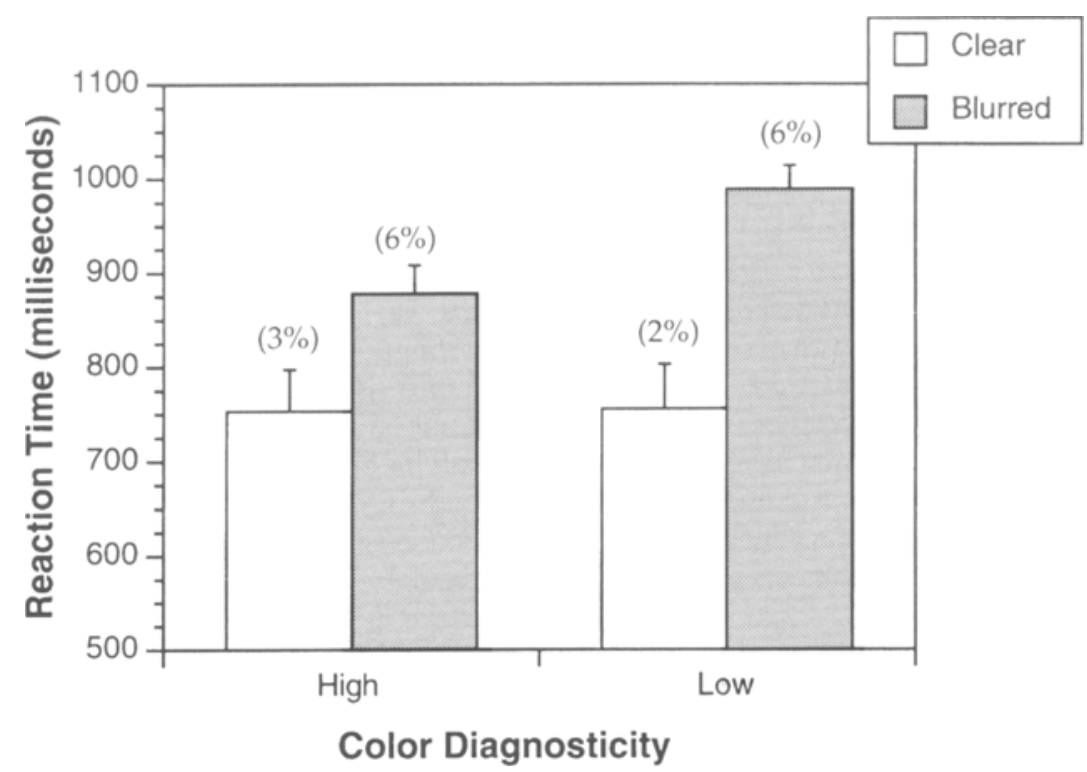

Figure 4. Mean reaction times for object verification (Experiment 5) as a function of color diagnosticity and clarity.

\begin{abstract}
Method
Subjects. The 35 subjects were students enrolled at Oberlin College. None of the subjects from the previous experiments participated in Experiment 5. All the subjects reported normal or corrected-tonormal vision and normal color vision. The subjects received class credit for their participation.

Stimuli. The stimuli were composed of the color versions of the 24 target item pictures ( 12 HCD and 12 LCD) and the 24 foil item pictures used in Experiment 2. These 48 items constituted the clear picture condition. Each target picture, as well as each foil picture, was then submitted to a Gaussian filter with a radius of 7 pixels, yielding an average object-to-filter pixel ratio of $20: 1$ in the horizontal dimension and a $16: 1$ object-to-filter pixel ratio in the vertical dimension (Parish \& Sperling, 1991). Each picture contained the object displayed against a neutral white background. The same object name labels as those employed in Experiment 2 were utilized in Experiment 5.

Procedure. The procedure for Experiment 5 was similar to that for the object classification task of Experiment 2. For each of the 48 exemplars, there were two clarity types (clear, blurred). Thus, there were 96 total trials. The presentation of trials was randomized across subjects.
\end{abstract}

\section{Results and Discussion}

Accuracy. An ANOVA was performed on the error data, with display (clear, blurred) and color diagnosticity (high, low) as within-subjects factors. There was a significant main effect of display $\left[F(1,34)=18.19, M S_{\mathrm{e}}=0.05\right.$, $p<.05]$. The subjects committed fewer errors when classifying pictures presented in the clear condition $(M=2 \%)$ than when classifying pictures in the blurred condition $(M=6 \%)$. The main effect of color diagnosticity testing differences between $\operatorname{HCD}(M=4 \%)$ and $\operatorname{LCD}(M=4 \%)$ objects was not significant $\left[F(1,34)=0.30, M S_{\mathrm{e}}=0.001\right.$, $p>.05]$, nor was the interaction between clarity and color diagnosticity significant $\left[F(1,34)=0.03, M S_{\mathrm{e}}=0.001\right.$, $p>$.05].
Reaction time. Reaction times were calculated for correct trials of HCD and LCD target objects (trials involving foil objects were omitted from further analyses). An ANOVA was performed, with display (clear, blurred) and color diagnosticity (high, low) as within-subjects factors. There was a significant difference between clear $(M=753 \mathrm{msec})$ and blurred $[M=932 \mathrm{msec} ; F(1,34)=$ 42.41, $\left.M S_{\mathrm{e}}=1,124,919, p<.05\right]$ displays. The subjects were faster to classify HCD pictures $(M=815 \mathrm{msec})$ than they were to classify LCD pictures $[M=871 \mathrm{msec}$; $\left.F(1,34)=7.63, M S_{\mathrm{e}}=111,060, p<.05\right]$. The interaction between clarity and color diagnosticity was also significant $\left[F(1,34)=8.41, M S_{\mathrm{e}}=106,826, p<.05\right]$. As is shown in Figure 4, although degrading the display significantly impaired classification, classification of LCD objects was more impaired under degraded shape conditions than was the classification of HCD objects, relative to their nondegraded conditions.

An ANOVA, with items as the random factor and display and color diagnosticity as within-items factors, showed that the difference in display was reliable $\left[F(1,22)=11.138, M S_{\mathrm{e}}=93,708, p<.01\right]$, but there was no overall effect of diagnosticity $\left[F(1,22)=1.361, M S_{\mathrm{e}}=\right.$ $31,646, p>.10]$. The interaction between diagnosticity and display was not significant $(p>.10)$. The individual reaction times for the HCD and LCD objects are listed in Appendix D.

The results from the subject analysis indicate that, when shape information is degraded, recognition of LCD objects is more impaired than recognition of HCD object. Although the item analysis suggests caution in interpretation, recognition of LCD objects seems to be dependent on the availability of shape information, whereas recognition of HCD objects relies on both color 
and shape information. It is important to emphasize that although shape degradation adversely affected the recognition of LCD objects, it also impaired recognition of HCD objects. Thus, it was not the case that the subjects were attending only to color information to make their classifications; they were also attending to object shape.

What is the advantage of representing objects in terms of the multiple dimensions of color and shape? As the results of Experiment 5 suggest, color-and-shape-represented objects might show an advantage over shape-only objects in conditions in which access to edge information is limited. For instance, given its distinctive yellow color, identifying a partially occluded banana should be easier than identifying a partially occluded can opener. The adaptive advantage of representing certain objects with respect to both color and shape information is that, under less than ideal viewing conditions, multicoded objects will suffer less than objects that are coded by a single dimension. It could be argued that, in the real world, recognition under occlusion is more the standard than the exception, and hence, color may play an important role in everyday object recognition.

\section{GENERAL DISCUSSION}

The general goal of these experiments was to test whether color information influences object recognition. According to the color diagnosticity hypothesis, color is more likely to affect recognition of objects that were high in color diagnosticity than recognition of objects that were low in color diagnosticity. In Experiment 1, HCD and LCD objects were identified, using a featurelisting task and typicality ratings. The effects of color information on recognition was demonstrated in Experiment 2 , in which the subjects were slower and less accurate to identify achromatic versions of HCD objects than to identify color versions. In contrast, the subjects showed no differences in errors or latencies when identifying achromatic and color versions of LCD objects. In Experiment 3, it was found that the subjects named color versions of HCD objects faster and with less error than achromatic versions. However, when the subjects named LCD objects, no differences between achromatic and color versions were found with respect to naming latencies or errors. In Experiment 4A, HCD and LCD objects were equated for their shape diagnosticity by matching their achromatic verification times. In Experiment 4B, the subjects demonstrated color facilitation and color interference effects when verifying HCD objects. In contrast, the subjects showed no evidence of color facilitation or color interference effects when verifying $L C D$ objects. Finally, in Experiment 5, in which shape information was degraded, the subjects showed less impairment in their recognition of visually degraded HCD objects than in their recognition of visually degraded LCD objects. Thus, consistent with the predictions of the color diagnosticity hypothesis, these results indicate that color plays a role in the recognition of objects with strong color associations.
The color effects obtained in the present study are at variance with the lack of color effects reported by other researchers (Biederman \& Ju, 1988; Ostergaard \& Davidoff, 1985). A closer examination of the objects used in previous experiments suggests some reasons for the discrepant results. Many of the objects identified as HCD on the basis of their color typicality by Biederman and Ju were judged as being only moderate or low in color diagnosticity by our subjects, using feature-listing and typicality responses. Color diagnosticity might depend not only on the typicality of the object's color, but also on its distinctiveness. Similar to other measures of cue validity (Wurm et al., 1993), the feature-listing method assesses the degree to which an object's color distinguishes it from other members of the object class. The color advantage in recognition might, therefore, depend on identifying HCD objects with the additional characteristic of color distinctiveness.

In another study, Ostergaard and Davidoff (1985) also found no difference between the recognition of consistently colored, inconsistently colored, and achromatic pictures of fruit (tomato, strawberry, radish). However, as was pointed out by Price and Humphreys (1989), color effects are more likely to be found in studies that use a wide range of stimulus objects of varying diagnostic colors. The relatively diverse set of HCD objects used in the present experiments may have contributed to the obtained color effects.

These experiments also address the relation between color diagnosticity and shape diagnosticity. In the limiting case, color would play an important role in recognition when objects are nearly identical in shape (Biederman \& Ju, 1988). For example, the color green would provide critical disambiguating information when trying to distinguish a frog from a toad. However, it is unlikely that the HCD objects described in these studies fall into the category of shape-identical objects. First, the selected HCD and LCD objects (e.g., banana, broccoli, dog, lamp) were basic level objects and, therefore, differentiable from other objects on the basis of their shape properties (Rosch et al., 1976). Second, the foil/distractor objects used in the object classification studies were selected specifically because they differed from the target objects with respect to their shape and color. Finally, in Experiment 4B, in which HCD and LCD objects were equated with respect to their shape diagnosticity, HCD objects nevertheless exhibited stronger color effects than did LCD objects. Collectively, these results suggest that the color effects were not an artifact of shape-identical objects.

Despite the positive evidence to support the influence of color in object recognition, the present results lead one to recommend that certain qualifications be applied to the color diagnosticity hypothesis. First, of the 10 HCD objects tested in recognition tasks involving classification, naming, and verification, only carrot, corn, and lemon were consistently identified more quickly in color than in gray scale. Hence, although color facilitated the recognition of HCD objects as a group, the color advantage for individual HCD objects fluctuated across ex- 
periments. Second, color effects were primarily restricted to the recognition of natural objects. Eight of the $12 \mathrm{HCD}$ objects were members of natural categories, whereas all of the LCD objects were members of artifactual categories. Objects from natural categories (e.g., fruit, animal, vegetables) tend to be structurally similar (Price \& Humphreys, 1989) and have prototypical colors (Tanaka \& Szechter, 1997). In contrast, objects from artifactual categories (e.g., tools, furniture, appliances) are more structurally divergent and generally lack characteristic colors. Although Experiment 4 attempted to isolate the effects of color diagnosticity from shape diagnosticity, color and shape most certainly interact in how humans learn to recognize objects from different categories. Given their less distinctive structural properties and more distinctive color properties, it seems more efficient for the human recognition system to weight color information more heavily when recognizing natural objects than when recognizing artifactual objects. As a consequence, color effects are more likely to be found in the recognition of natural objects than in the recognition of human-made objects. Thus, the results from these experiments indicate that color effects are sensitive to the recognition paradigm employed and are primarily restricted to the recognition of natural objects.

Although, in our experiments, we have classified stimulus objects as being either high or low in color diagnosticity, color diagnosticity is probably better described as a continuum, with objects with strong color associations lying on one end of the continuum, objects with no color associations on the other end, and objects with moderate color associations lying somewhere in between. The extent to which color information contributes to object recognition would depend on where the object lies along the color diagnosticity continuum. For objects that are high in color diagnosticity, color would be weighted more heavily in recognition; for objects that are low in color diagnosticity, color would play little or no role in recognition. The idea that modality-specific features can be differentially weighted according to their diagnosticity is compatible with present parallel distributed models of object categorization (Farah \& McClelland, 1991).

In summary, our results show that color information can exert an influence on object recognition stage processes. The consistent finding of the reported experiments is that the presence or the absence of color affects the recognition of HCD, but not of LCD objects. Thus, these results indicate that the object recognition system is not completely colorblind when it comes to the identification of HCD objects.

\section{REFERENCES}

Battig, W. F., \& Montague. W. E. (1969). Category norms for verbal items in 56 categories: A replication and extension of the Connecti- cut category norms. Journal of Experimental Psychology Monographs, 80(3, Pt. 2).

BIEDERMAN, I. (1987). Recognition-by-components: A theory of human image understanding. Psychological Review, 94, 115-147.

Biederman, I., \& Ju, G. (1988). Surface versus edge-based determinants of visual recognition. Cognitive Psychology, 20, 38-64.

Callaghan, T. C. (1984). Dimensional interaction of hue and brightness in preattentive field segregation. Perception \& Psychophysics, 36, 25-34.

CavanaGH, P. (1987). Reconstructing the third dimension: Interactions between color, texture, motion, binocular disparity, and shape. Computer Vision, Graphics \& Image Processing, 37, 171-195.

Corbeil, J. C., \& Archambault, A. (Eds.) (1992). The MacMillan visual dictionary. New York: MacMillan.

DavidofF. J. B., \& OstergaARd, A. L. (1988). The role of color in categorical judgments. Quarterly Journal of Experimental Psychology, 40A 533-544.

FARAH, M. J., \& MCCLELLAND, J. L. (1991). A computational model of semantic memory impairment: Modality specificity and emergent category specificity. Journal of Experimental Psychology: General, 120, 339-357.

GiBson, E. J. (1969). Principles of perceptual learning and development. New York: Appleton-Century-Crofts.

GrossberG, S., \& Mingolla, E. (1985). Neural dynamics of perceptual grouping: Textures, boundaries, and emergent segmentations. Perception \& Psychophysics, 38, 141-171.

humphrey, G. K., Goodale, M. A., Jakobson, L. S., \& Servos, P. (1994), The role of surface information in object recognition: Studies of visual form agnosic and normal subjects. Perception, 23, 1457 1481

Joseph, J. E., \& ProffitT, D. (1996). Semantic versus perceptual influences of color in object recognition. Journal of Experimental Psychology: Learning, Memory, \& Cognition, 22, 407-429.

Legge, G. E., Parish, D. H., Luebker, A., \& Wurm, L. H. (1990). Psychophysics of reading: XI. Comparing color contrast and luminance contrast. Journal of the Optical Society of America A, 7, 2002 2010.

Livingstone, M. D., \& Hubel. D. H. (1987). Psychophysical evidence for separate channels for the perception of form, color, movement, and depth. Journal of Neuroscience, 7, 3416-3468.

MAPELL, D.. \& BehrmanN, M. (1997). The role of color in object recognition: Evidence from visual agnosia. Neurocase, 3, 237-247.

MARR, D. (1982). Vision. San Francisco: Freeman.

Neisser, U. (1967). Cognirive psychology. New York: AppletonCentury-Crofts.

Ostergaard, A. L., \& Davidoff, J. B. (1985). Some effects of color on naming and recognition of objects. Journal of Experimental Psychology: Learning. Memory, \& Cognition, 11, 579-587.

PARISH. D. H., \& SPERLING, G. (1991). Object spatial frequencies, retinal spatial frequencies, noise, and the efficiency of letter discrimination. Vision Research, 31, 1399-1415.

Price, C. J., \& Humphreys, G. W. (1989). The effects of surface detail on object categorization and naming. Quarterly Journal of Experimental Psychology, 41 A, 797-828.

Rosch. E., Mervis. C. B., Gray, W., Johnson. D., \& Boyes-Braem, P. (1976). Basic objects in natural categories. Cognitive Psychology, 8 , 382-439.

TANAKa, J. W.. \& Szechter. L. (1997). Normative feature listings for the 260 Snodgrass and Vanderwart objects. Unpublished manuscript.

Troscianko, T., \& HarRIs, J. P. (1988). Phase discrimination in compound chromatic gratings. Vision Research, 28, 1041-1049.

Ullman, S. (1984). Visual routines. Cognition, 18, 97-159.

Wurm, L. H., LegGe, G. E., Isenberg, L. M., \& Luebker. A. (1993). Color improves object recognition in normal and low vision. Journal of Experimental Psychologv: Human Perception \& Performance, 19. 899-911. 
APPENDIX A

Feature-Listing Items (Experiment 1)

\begin{tabular}{|c|c|c|c|c|c|c|c|}
\hline Item & Typical Color & $\%$ Agreement & $\%$ First Mention & Item & Typical Color & $\%$ Agreement & $\%$ First Mention \\
\hline Apple & red & 97 & 53 & Lamp & white & 53 & 7 \\
\hline Banana & yellow & 100 & 83 & Lemon & yellow & 100 & 90 \\
\hline Baseball & white & 93 & 37 & Lettuce & green & 97 & 83 \\
\hline Basketball & orange & 77 & 27 & Lime & green & 100 & 87 \\
\hline Bee & yellow & 73 & 30 & Nail & silver & 83 & 10 \\
\hline Bird & blue/brown & 23 & 3 & Pea & green & $: 00$ & 40 \\
\hline Brick & red & 87 & 80 & Pear & yellow & 53 & 33 \\
\hline Broccoli & green & 100 & 83 & Pencil & yellow & 97 & 27 \\
\hline Camera & black & 100 & 53 & Penny & brown & 93 & 47 \\
\hline Carrot & orange & 100 & 80 & Pig & pink & 97 & 67 \\
\hline Chair & brown & 90 & 0 & Potato & brown & 97 & 40 \\
\hline Cigar & brown & 97 & 47 & Radish & red & 83 & 83 \\
\hline Corn & yellow & 100 & 80 & Saw & silver & 77 & 3 \\
\hline Cow & white & 47 & 40 & Schoolbus & yellow & 100 & 70 \\
\hline Dog & brown & 80 & 0 & Screwdriver & silver & 83 & 13 \\
\hline Donkey & brown & 57 & 47 & Sportscar & red & 100 & 26 \\
\hline Fire engine & red & 100 & 97 & Stop sign & red & 100 & 80 \\
\hline Fish & silver & 50 & 3 & Strawberry & red & 100 & 77 \\
\hline Flowerpot & brown & 43 & 27 & Table & brown & 100 & 0 \\
\hline Football & brown & 93 & 50 & Taxi & yellow & 100 & 97 \\
\hline Fork & gray & 100 & 23 & Tennis ball & yellow & 60 & 37 \\
\hline Grape & purple & 60 & 27 & Tiger & orange & 57 & 27 \\
\hline Hammer & gray & 60 & 3 & Tomato & red & 97 & 77 \\
\hline Horse & brown & 97 & 43 & Yield sign & yellow & 80 & 60 \\
\hline
\end{tabular}

APPENDIX B

Target Objects and Foil Objects for Experiment 2

\begin{tabular}{ll}
\hline \multicolumn{1}{c}{ Target Objects } & Foil Objects \\
Radish & Oolor Diagnosticity \\
Lime & Onion \\
Lemon & Peach \\
Carrot & Orange \\
Taxi & Pepper \\
Fire engine & Limousine \\
Broccoli & Tractor \\
Lettuce & Cauliflower \\
Corn & Eggplant \\
Stop sign & Celery \\
Banana & Merge sign \\
Brick & Cucumber \\
& Matchbox \\
Chair & Low Color Diagnosticity \\
Lamp & Sofa \\
Table & Umbrella \\
Bird & Chest \\
Nail & Bat \\
Fork & Screw \\
Dog & Rake \\
Hammer & Horse \\
Fish & Ax \\
Screwdriver & Dolphin \\
Saw & Wrench \\
Sportscar & Knife \\
& Stationwagon \\
\hline & \\
&
\end{tabular}

APPENDIX C

Space-Averaged Luminance $\left(\mathrm{cd} / \mathrm{m}^{2}\right)$ Values for High and Low Color Diagnostic Objects

\begin{tabular}{lcr}
\hline \multicolumn{1}{c}{ Objects } & Gray Scale & \multicolumn{1}{c}{ Color } \\
\hline & High Color Diagnostic \\
Banana & 22.40 & 23.00 \\
Broccoli & 7.26 & 7.56 \\
Brick & 11.09 & 10.81 \\
Carrot & 12.00 & 11.03 \\
Corn & 15.50 & 17.23 \\
Fire engine & 9.45 & 9.49 \\
Lemon & 18.80 & 17.90 \\
Lettuce & 8.02 & 8.92 \\
Lime & 5.74 & 5.77 \\
Radish & 8.47 & 8.37 \\
Stop sign & 7.97 & 7.95 \\
Taxi & 23.00 & 25.50
\end{tabular}

Low Color Diagnostic

$\begin{array}{lrr}\text { Bird } & 6.24 & 7.63 \\ \text { Chair } & 1.87 & 2.17 \\ \text { Dog } & 9.36 & 9.40 \\ \text { Fish } & 3.04 & 3.22 \\ \text { Fork } & 22.60 & 22.20 \\ \text { Hammer } & 24.10 & 24.70 \\ \text { Lamp } & 26.10 & 26.00 \\ \text { Nail } & 6.24 & 7.63 \\ \text { Saw } & 18.10 & 18.57 \\ \text { Screwdriver } & 16.65 & 16.56 \\ \text { Sports car } & 10.71 & 10.59 \\ \text { Table } & 8.71 & 8.82\end{array}$


APPENDIX D

Mean Reaction Times (in Milliseconds) for Target Items as a Function of Color Diagnosticity and Display for Experiments $2,3,4 \mathrm{~B}$, and 5

\begin{tabular}{|c|c|c|c|c|c|c|c|c|c|}
\hline \multirow[b]{2}{*}{ Objects } & \multicolumn{2}{|c|}{$\begin{array}{l}\text { Experiment } 2 \\
\text { Classification }\end{array}$} & \multicolumn{2}{|c|}{$\begin{array}{l}\text { Experiment } 3 \\
\text { Naming }\end{array}$} & \multicolumn{3}{|c|}{$\begin{array}{l}\text { Experiment 4B } \\
\text { Verification }\end{array}$} & \multicolumn{2}{|c|}{$\begin{array}{c}\text { Experiment } 5 \\
\text { Degraded Shape }\end{array}$} \\
\hline & Achromatic & Color & Achromatic & Color & Achromatic & Congruent & Incongruent & Clear & Blurred \\
\hline \multicolumn{10}{|c|}{ High Color Diagnostic } \\
\hline Apple & & & & & 627 & 522 & 650 & & \\
\hline Banana & 663 & 620 & 634 & 648 & 620 & 564 & 619 & 593 & 526 \\
\hline Brick & 740 & 701 & 678 & 702 & 582 & 591 & 680 & 651 & 823 \\
\hline Broccoli & 793 & 792 & 764 & 747 & 621 & 612 & 669 & 640 & 597 \\
\hline Carrot & 747 & 664 & 772 & 633 & 611 & 544 & 751 & 614 & 479 \\
\hline Celery & & & & & 762 & 814 & 713 & & \\
\hline Corn & 752 & 655 & 821 & 709 & 628 & 596 & 722 & 709 & 889 \\
\hline Fire engine & 762 & 725 & $77 !$ & 786 & 693 & 556 & 775 & 585 & 932 \\
\hline Lemon & 1,049 & 770 & 904 & 733 & 737 & 580 & 806 & 624 & 508 \\
\hline Lettuce & 1,028 & 868 & 792 & 864 & & & & 688 & 1,513 \\
\hline Lime & 1,512 & 1,212 & 1,022 & 864 & & & & 1,032 & 717 \\
\hline Radish & 915 & 758 & 972 & 823 & 678 & 721 & 906 & 592 & 636 \\
\hline Stop sign & 581 & 576 & 680 & 696 & 604 & 639 & 591 & 388 & 623 \\
\hline Taxi & 751 & 722 & 854 & 926 & 844 & 613 & 742 & 479 & 494 \\
\hline \multicolumn{10}{|c|}{ Low Color Diagnostic } \\
\hline Bee & & & & & 732 & 631 & 789 & & \\
\hline Bird & 713 & 701 & 673 & 667 & 604 & 531 & 584 & 721 & 689 \\
\hline Chair & 745 & 789 & 632 & 619 & 550 & 600 & 614 & 617 & 665 \\
\hline Crab & & & & & 610 & 636 & 641 & & \\
\hline Dog & 677 & 669 & 603 & 621 & & & & 524 & 722 \\
\hline Eggbeater & & & & & 627 & 733 & 763 & & \\
\hline Fish & 647 & 715 & 645 & 710 & 623 & 604 & 620 & 618 & 704 \\
\hline Fork & 662 & 712 & 645 & 710 & & & & 469 & 926 \\
\hline Forklift & & & & & 652 & 733 & 763 & & \\
\hline Hammer & 840 & 854 & 647 & 674 & & & & 561 & 1,047 \\
\hline Lamp & 735 & 768 & 716 & 707 & 568 & 536 & 607 & 610 & 701 \\
\hline Nail & 817 & 899 & 667 & 679 & & & & 629 & 705 \\
\hline Paint brush & & & & & 655 & 701 & 587 & & \\
\hline Saw & 838 & 769 & 675 & 676 & 685 & 602 & 708 & 718 & 781 \\
\hline Screwdriver & 1,049 & 926 & 724 & 743 & 703 & 653 & 661 & 552 & 697 \\
\hline Sportscar & 737 & 690 & 784 & 802 & & & & 667 & 679 \\
\hline Table & 737 & 693 & 756 & 723 & & & & 586 & 519 \\
\hline Tape dispenser & & & & & 622 & 607 & 608 & & \\
\hline
\end{tabular}

(Manuscript received May 22, 1995; revision accepted for publication June 24, 1998.) 